\title{
Medición de Impedancia Eléctrica en Tejido Biológico - Revisión
}

\author{
María E. Moncada ${ }^{1}$ \\ María del P. Saldarriaga ${ }^{2}$ \\ Andrés F. Bravo ${ }^{3}$ \\ Carlos R. Pinedo 4
}

\section{Resumen}

El siguiente trabajo presenta una revisión de las propiedades eléctricas de los tejidos biológicos. Los tópicos incluidos en el trabajo son el estudio, caracterización y técnicas de medición. Se aborda el tema de las propiedades de conductividad ( $\sigma$ ) y permitividad (ع) eléctrica de los tejidos y los modelos creados para su estimación y aproximación. Se presentan las características de los elementos de medición, ventajas y desventajas de los diferentes métodos creados y sus aplicaciones. Se mencionan algunos de los tipos de señales más utilizadas en cuanto a corriente y frecuencia de aplicación. Finalmente se presentan algunas recomendaciones a tener en cuenta al momento de realizar mediciones in vivo, para disminuir los errores debido a las múltiples fuentes fisiológicas. Esta revisión permitió obtener los lineamientos y condiciones de un trabajo de medición de impedancia de fémur que se encuentra en desarrollo.

1 Centro de Investigación, INSTITUTO TECNOLÓGICO METROPOLITANO, mariamoncada@itm.edu.co

2 Facultad de Ingeniería, Escuela de ingeniería Eléctrica y Electrónica, Universidad del Valle, pilarcita87@hotmail.com

3 Facultad de Ingeniería, Escuela de ingeniería Eléctrica y Electrónica, Universidad del Valle, afbrandbravo@hotmail.com

4 Facultad de Ingeniería, Escuela de ingeniería Eléctrica y Electrónica, Universidad del Valle, carlos.pinedo@correounivalle.edu.co

Fecha de recepción: 14 de Julio de 2010

Fecha de aceptación: 13 de Octubre de 2010

Rev. Tecno Lógicas No. 25, ISSN 0123-7799, Diciembre de 2010, pp. 51-76 


\section{Palabras clave}

Bioimpedancia, medición eléctrica, propiedades eléctricas, tejido biológico.

\section{Abstract}

This paper presents a review of the electrical properties of biological tissues regarding their study, characterization, and measurement techniques. Electrical conductivity ( $\sigma)$ and permittivity ( $\varepsilon$ property of tissues, and models developed for their estimation and approximation are mentioned. Measurement elements, advantages and disadvantages of different methods and their applications are presented. Some types of signals used in terms of current and frequency of application are described. Finally, some recommendations to consider for measurements in vivo to reduce errors produced by multiple physiological sources are done. This review permitted to obtain guidelines and conditions for impedance measurement work of femur, which one is doing.

\section{Keywords}

Bioimpedance, biological tissue, electrical measurement, electrical properties. 


\section{INTRODUCCIÓN}

Los métodos para la medición de impedancia eléctrica en tejido biológico han sido investigados por muchos años en áreas médicas y biológicas aplicadas (Jossinet, 1988; Geddes et al., 1990; Gabriel \& Gabriel, 1996; Martinsen et al., 2000; Salazar et al., 2004; Gonzalez et al., 2006; 2007; Katz et al., 2006; Moncada et. al, 2008). Una evidencia del esfuerzo e interés en esta área se representa en la existencia de un Comité Internacional para la Promoción de la Investigación en Bioimpedancia (ICPRBI), el cual ha impulsado la realización de once Conferencias Internacionales sobre Bioimpedancia Eléctrica entre 1969 (New York) y 2001 (Oslo). La mayoría de las investigaciones y publicaciones han sido dirigidas principalmente al desarrollo de protocolos de medida adecuados, el uso de electrodos de contacto, mejorar la instrumentación y a la mejor interpretación de los resultados (Salazar, 2004). En Colombia se han desarrollado algunos trabajos de medición de bioimpedancia en muestras animales (Gonzalez, et al., 1997, Moncada et al., 2008) y en pacientes (González et al., 2007).

El método de medición de propiedades eléctricas con bioimpedancia es de bajo costo, de medición rápida y presenta aplicación para muchas partes del cuerpo. La baja exactitud reportada (al compararlo con el gold estándar) ha reducido la credibilidad del método. Sin embargo, recientes investigaciones han mostrado por ejemplo, que el método de espectroscopia de bioimpedancia puede ser tan precisa como los métodos de dilución tradicionales y aún mejor (Medrano, 2007).

Una de las técnicas más prominentes en el área de mediciones de bioimpedancia es la medición no invasiva por medio de electrodos (Salazar, 2004), la de corrientes inducidas por campos magnéticos (Hagmann, 1993; Gandhi \& Chen, 1990; Blackwell, 1990) y los métodos híbridos que combinan las dos técnicas (Gencer et al., 1994; Zlochiver et al., 2002). Estos métodos proporcionan información sobre las características eléctricas de bioimpedancia y sus cambios ante patologías, sin invadir los tejidos. De igual forma, la gran variedad de tejidos y sus particulares propiedades eléctricas y dieléctricas hacen de la 
bioimpedancia una técnica para el monitoreo de imagen del cuerpo y sus partes (Paavle et al., 2007).

Gracias a los estudios en propiedades eléctricas se han logrado desarrollar diferentes métodos de medición de bioimpedancia (Salazar, 2004; Rafiei et al., 2007; Hagmann, 1993), se ha logrado mejorar y desarrollar electrodos de diferentes características (Geddes \& Ragheb, 1990; Geddes, 1997), construir equipos cada vez más precisos (Northrop, 2002; Zhu et al., 2007), mejorar los procedimientos de preparación de las muestras y pacientes (Slinde \& Rossander, 2001; Slinde et al., 2003; Medrano, 2006; Medrano et al., 2007) y crear modelos matemáticos y computacionales para la estimación de las variables de medida (Zlochiver et al., 2002; 2005).

Toda la evidencia y aplicación encontrada en el desarrollo de las mediciones de bioimpedancia, hacen de esta área un área en continua formación y aún con mucho por explorar e investigar. Nuevos métodos no invasivos, mejoramiento en las características de los electrodos, desarrollos matemáticos y más estudio de los métodos sin contacto, son solo algunas de las temáticas que pueden llegar a fortalecer el diagnóstico y tratamiento de patologías para diferentes partes del cuerpo.

\section{PROPIEDADES DIELÉCTRICAS DEL TEJIDO BIOLÓGICO}

Cuando un dieléctrico se expone a un campo eléctrico se generan en él procesos químicos y físicos. La representación eléctrica de este comportamiento puede ser descrita mediante dos propiedades principales la conductividad ( $\sigma$ ) y la permitividad (ع) eléctricas. La conductividad y permitividad eléctrica son cantidades tensoriales, pero pueden ser consideradas en medio isotrópicos y con respuesta independiente del tiempo, lo que lleva a simplificarlas como valores escalares dependientes de la frecuencia y que pueden ser expresados como un número complejo (Rigaud et al., 1996).

La célula es una estructura compleja constituida por una membrana cerrada que exhibe un comportamiento "inteligente" (semipermeable y selectivo) formada básicamente por una doble 
capa de lípidos con proteínas. Las diversas moléculas que se encuentran "insertadas" en ellas poseen diferentes afinidades y fobias, en especial al agua (Rigaud et al., 1996). Cuando se agrupan células con una función específica se forman los tejidos, quedando entre ellas espacios llenos del líquido extracelular. Cada una de esas estructuras y regiones poseen propiedades eléctricas muy particulares que dependiendo de su constitución y estado, determinarán la conducta de las propiedades eléctricas en función de la frecuencia (Foster \& Schwan, 1996).

Cuando el tejido se encuentra bajo la acción de un campo eléctrico, en él se inducen fuerzas actuantes que llevan a la polarización de estructuras no polares o a la orientación de los dipolos ya existentes. La respuesta de una muestra biológica a la acción del campo eléctrico dependerá de las características de sus estructuras y dipolos, así como su capacidad de formación y orientación.

En las diferentes regiones del tejido biológico se han identificado tres factores de relajación o dispersión relacionados con la respuesta del tejido a la corriente y la frecuencia. Para bajas frecuencias (inferior a cientos de $\mathrm{kHz}$ ) la conductividad de los tejidos es dominada por conducción de electrolitos en el espacio extracelular. Los tejidos presentan la dispersión alfa (a) debido a procesos físicos que incluye la polarización a lo largo de la estructura de frontera de la membrana. A frecuencias inferiores a la dispersión alfa, la permitividad relativa del tejido alcanza valores muy altos (decenas de millón) haciéndose más notable en la conductividad que en la permitividad. A radiofrecuencias (entre $0.1 \mathrm{MHz}$ y $10 \mathrm{MHz}$ ) los tejidos presentan la dispersión beta (B), en esta, la membrana celular tiene impedancia despreciable y la corriente pasa a través del medio intracelular y extracelular manifiesto en la permitividad y conductividad. A frecuencias de microondas (sobre $1 \mathrm{GHz}$ ) los tejidos presentan la dispersión gamma (Y) debido a la relación rotacional del tejido en el agua. Esta dispersión es centrada a $20 \mathrm{GHz}$ y es la misma que se encuentra en el líquido de agua (Grimnes \& Martinsen, 2000). La Fig. 1. muestra las frecuencias de dispersión para el tejido biológico en función de la frecuencia. 


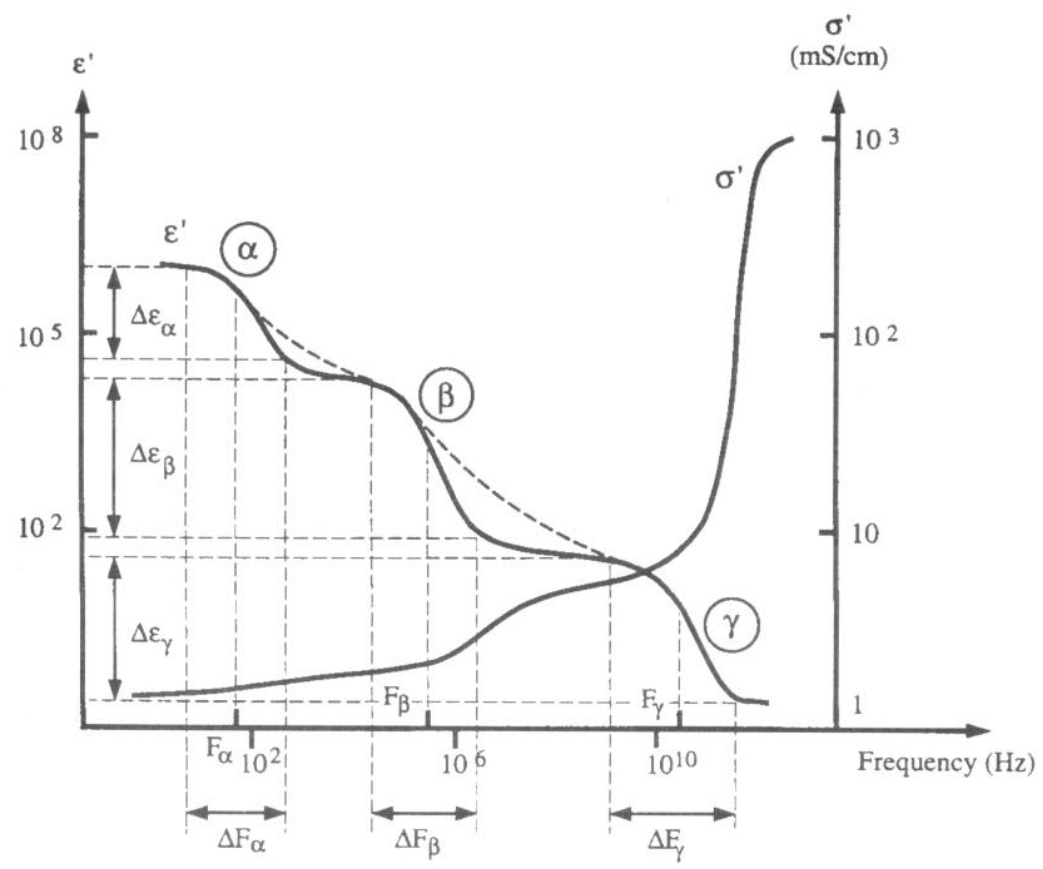

Fig. 1. Conductividad $\sigma$ y $\varepsilon$ en función de la frecuencia (Bourne et al., 1996)

\section{MEDICIÓN DE CONDUCTIVIDAD Y PERMITIVIDAD DE TEJIDOS BIOLÓGICOS}

Una forma simple de medición de las propiedades dieléctricas de permitividad y conductividad de una muestra desconocida, puede ser a partir de la medición de una impedancia de prueba contra una muestra conocida usando (1) y (2) desarrolladas por Gabriel et al., (1996 a, b):

$$
\begin{aligned}
\varepsilon^{\prime} & =\frac{C}{k} \\
\sigma & =\frac{G \varepsilon_{0}}{k}
\end{aligned}
$$


Los datos eléctricos de los tejidos pueden ser representados por varios caminos. La literatura orientada a la fisiología, presentan frecuentemente las propiedades dieléctricas de los tejidos en series de formas equivalentes (como impedancia de tejido) más que en la forma de equivalente paralelo (permitividad compleja). Sin embargo, el proceso fundamental relacionado con el aumento de la densidad de carga y la conducción eléctrica ocurren en paralelo y las propiedades eléctricas de un material son presentadas en su mayoría como combinaciones paralelo de un capacitor (permitividad) y un elemento conductor (conductividad).

Uno de los estudios más completos en medición de tejidos biológicos es el desarrollado por Gabriel et al., (1996 a, b). En su estudio fueron tomadas mediciones múltiples sobre muestras estándares de composición uniforme. Las propiedades de conductividad y permitividad reportadas fueron para más de 30 tipos de tejidos diferentes. El error reportado de los valores fue de $\pm 5 \%$ para frecuencias superiores a $100 \mathrm{MHz}$ y $\pm 15 \%$ para bajas frecuencias.

Para frecuencias inferiores a $100 \mathrm{~Hz}$ la contribución de la componente capacitiva en la impedancia de los materiales biológicos es solo del orden de $10 \%$ en la mayoría de los casos. Por lo que en la práctica, para bajas frecuencias, son consideradas condiciones cuasi-estáticas, donde no es considerado el valor de la permitividad.

Los tejidos biológicos son medios heterogéneos que involucran diversas constantes de tiempo, la función más utilizada para representar esta situación es la denominada ecuación de permitividad de Cole-Cole (3) (Curtze et al., 2004):

$\varepsilon^{*}(\omega)=\varepsilon_{\alpha}+\frac{\varepsilon_{s}+\varepsilon_{\alpha}}{1+j \omega\left(\tau_{c}\right)(1-\alpha)}-\frac{j \sigma_{s}}{\omega \varepsilon_{0}}$

Donde a es un parámetro empírico característico de la distribución de la frecuencia de relajación $f_{c}\left(\tau_{c} \frac{1}{2 \pi f_{c}}\right)$, dando una idea del grado de dispersión de las constantes de tiempo asociadas con el fenómeno de relajación. De esta forma, (4) encuentra una 
expresión análoga para la impedancia. La representación del modelo eléctrico y las ecuaciones características se muestran en la Fig. 2 (Curtze et al., 2004).

$Z=R_{\alpha}+\frac{R_{0}-R_{\alpha}}{1+j \omega\left(\tau_{c}\right)(1-\alpha)}$

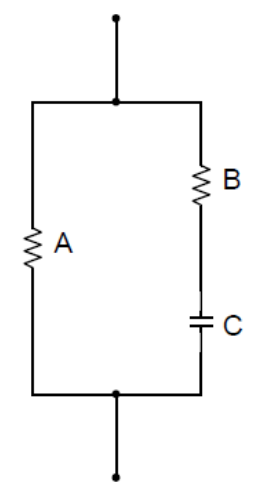

$$
\begin{aligned}
& A=R_{0} ; B=\frac{R_{0} R_{\infty}}{R_{0}-R_{\infty}} ; \\
& C=-j\left(\frac{R_{0}^{2}}{R_{0}-R_{\infty}}\right)\left(\frac{f_{c}}{f}\right)^{(1-\alpha)}
\end{aligned}
$$

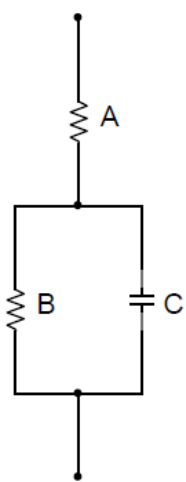

$A=R_{\infty} ; B=\Delta R=R_{0}-R_{\infty} ;$

$C=-j \Delta R\left(\frac{f_{c}}{f}\right)^{(1-\alpha)}$

Fig. 2. Circuito eléctrico del modelo de Cole-Cole para un solo arco (Kun, \& Peura, 1999)

La variación de la impedancia en los tejidos biológicos puede ser graficada como el cambio de la reactancia compleja en función de la resistencia. La Fig. 3 representa el lugar geométrico de Nyquist, que para circuitos RC simples como los que resultan del modelo, produce un semicírculo con centro en el eje real de la impedancia. La frecuencia aumenta de derecha a izquierda, desde $R_{0}$ hasta $R_{\infty}$. La depresión del centro del arco es expresada por el ángulo a. Los datos que se obtienen $\mathrm{Pi}(\mathrm{xi}$, yi) están sobre el semicírculo. 
En la Fig. 3 pueden observarse dos intersecciones en el eje real de la impedancia; $R_{0}$ (representando la resistencia en DC) y en $R_{\infty}$ (representando la resistencia para una frecuencia infinita). Es necesario entonces calcular los parámetros $R_{0}$, $R_{\infty}$, a y $\tau$ que provean la relación que mejor muestre o cuantifique la impedancia del tejido. El modelo de Cole-Cole es detallado en las siguientes referencias (Martinsen et al., 2000; Rigaud et al., 1996).

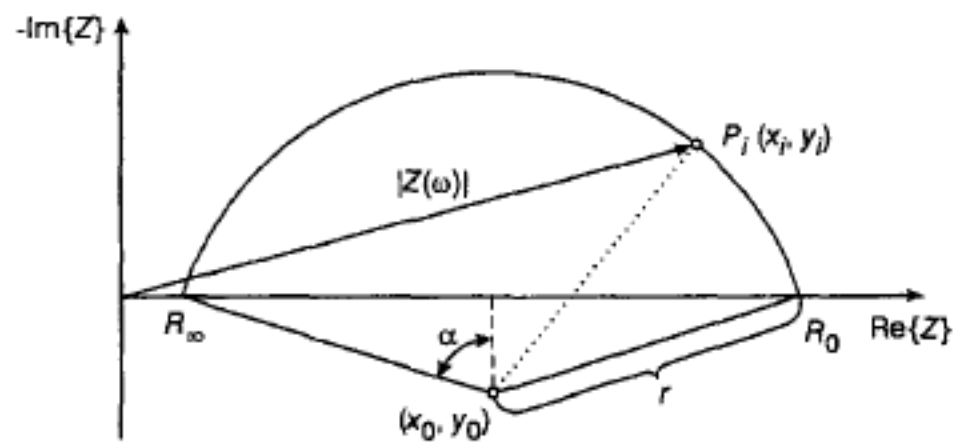

Fig. 3. Gráfico de la reactancia negativa del tejido $(-\mathrm{X}=-\operatorname{Im}\{\mathrm{Z}\})$ a todas las frecuencias forma el lugar geométrico (Kun, \& Peura, 1999)

\section{TEORÍA DE ELECTRODOS}

Los electrodos son los elementos transductores entre la corriente eléctrica del equipo de medida y la corriente iónica del tejido biológico. Una de las principales características es que deben presentar muy baja impedancia de entrada para reducir las tensiones parásitas inducidas. Los electrodos más usados para la medición de fenómenos bioeléctricos son tres: a) micro-electrodos, utilizados para medir potenciales bioeléctricos cerca o dentro de una célula; b) electrodos superficiales, utilizados para medir potenciales en la superficie de la piel, usados en electrocardiograma (ECG), electroencefalograma (EEG) y electro miografía (EMG) y c) electrodos de aguja, estos atraviesan la piel para el registro de potencial y son usados para mediciones EEG o musculares específicas. 


\subsection{Electrodos y Tejido Biológico}

La interface entre el contacto metálico de los electrodos y las diferentes disoluciones da lugar a un potencial eléctrico denominado potencial de electrodo. Para alcanzar el equilibrio se forma una capa en la interfaz, en realidad una doble capa, donde la capa próxima a la disolución presenta polaridad opuesta a la capa próxima al metal. En las mediciones bioeléctricas el potencial del electrodo se produce entre la interface del metal y un electrolito.

La doble capa de interface actúa como condensador, la Fig. 4 muestra el circuito equivalente del electrodo para biopotenciales en contacto con el cuerpo (voltaje en serie con una red de resistencias y condensador) (Ragheb \& Geddes 1990; Geddes, 1997). Dado que las medidas de los potenciales bioeléctricos requieren dos electrodos, la tensión medida es en realidad la diferencia entre los potenciales instantáneos de ambos.

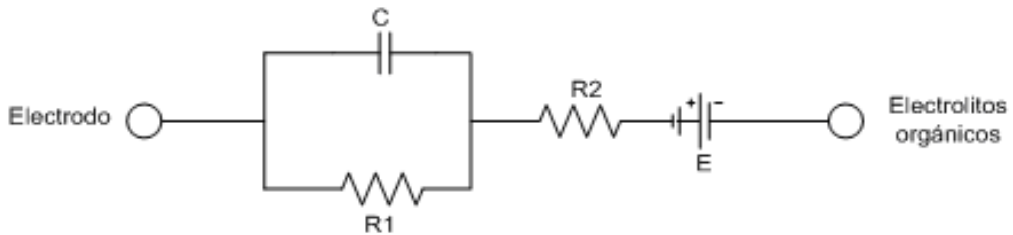

Fig. 4. Circuito equivalente de electrodo para biopotencial (Ragheb \& Geddes 1990)

La Fig. 4 muestra la representación de la impedancia de los electrodos (una de sus características más importantes) como una red resistencia-condensador de valores fijos de resistencia y capacitancia. Sin embargo, en la realidad estos varían con la frecuencia debido al efecto de polarización.

Cuando los electrodos difieren entre sí se produce una tensión conocida como tensión de offset, generando un flujo de corriente y provocando fluctuaciones en la tensión medida sin ninguna entrada fisiológica (provocando error en la medición). Este fenómeno puede reducirse seleccionando materiales adecuados y/o realizando un recubrimiento especial con métodos electrolíticos que mejoren su estabilidad. 


\subsection{Polarización de Electrodos}

El fenómeno de polarización es una manifestación de la organización de cargas moleculares en la interface electrodoelectrolito en presencia de agua, moléculas e iones hidratados. Su efecto incrementa al incrementar la conductividad de la muestra y sus consecuencias son más pronunciadas en la capacitancia que en la conductancia, tanto para soluciones iónicas como para muestras biológicas (Foster \& Schwan, 1996).

Cuando la medición se realiza sobre muestras biológicas, la mala conducción de las células apantalla parte del electrodo de allí que el material del electrodo es fundamental para determinar la impedancia de polarización. La inductancia de la prueba y el cable de conexión adicionan otra serie de componentes que interfieren con la medida. El tamaño y el tipo de electrodo también son importantes en la determinación de su impedancia, los más grandes (electrodos superficiales) tienden a tener impedancias más pequeñas, mientras los más pequeños (electrodos de aguja $o$ micro-electrodos) tienen impedancias de magnitud mucho mayor (Geddes, 1997).

\section{MEDICIÓN DE BIOIMPEDANCIA}

La metodología o técnica más común para el registro de las propiedades y señales eléctricas de los tejidos biológicos, utiliza electrodos de contacto para la inyección de una corriente eléctrica y otros dos electrodos sobre la región de interés para la medición de la caída de potencial (Salazar, 2004; Bragos et al., 1999; Rafiei et al., 2007; Beckmann et al., 2007). Otra de las formas, aunque menos explorada, es la medición a través de inducción magnética (Hagmann, 1993; Gandhi \& Chen, 1990) donde un campo magnético primario es aplicado al sitio de interés y es leído un campo magnético secundario. El valor se obtiene al obtener las diferencias entre el campo primario y el secundario. 


\subsection{Métodos con Contacto}

Las técnicas tradicionalmente utilizadas para la medida de la impedancia eléctrica de tejido biológico para bajas frecuencias se basan en: (a) la aplicación de una tensión conocida y la posterior medida de la corriente que se establece en la muestra, (b) la inyección de una corriente conocida y la posterior medida de la caída de potencial que se produce entre dos puntos escogidos o (c) la medida de ambas magnitudes físicas. Tanto la entrada como la salida de señales se realizan a través de electrodos que se encuentran en contacto físico. Los sistemas de medida pueden estar diseñados para trabajar con 2 ó más electrodos. La técnica más comúnmente utilizada es la b) a desarrollarse mediante: el método de 2 electrodos o bipolar, el método de 4 electrodos o tetrapolar y el métodos de tres electrodos (Salazar, 2004).

El método de los 2 electrodos, hace circular una corriente constante $\left(\mathrm{I}_{\mathrm{o}}\right)$ a través de dos electrodos con impedancia $\mathrm{Z}_{\mathrm{e}}$ (mayor que la impedancia a medir) y se obtiene el voltaje $\mathrm{V}_{\mathrm{o}}$ (función de la impedancia entre electrodos y los cambios fisiológicos). La impedancia medida la relaciona (5):

$Z=\frac{V_{0}}{I_{0}}=Z_{e 1}+Z_{e 2}+Z_{e 3}$

$\mathrm{Si}$ se considera que los electrodos tienen las mismas características $\left(Z_{e 1}+Z_{e 2}\right)$ y que se usan electrodos superficiales, donde la impedancia de la piel esta en serie con la impedancia del electrodo, la ecuación (5) se reduce. Sin embargo para obtener información de un tejido en particular no puede ser considerada la impedancia de la piel. Este requerimiento llevo al desarrollo del método de medición a 4 electrodos.

El método de los 4 electrodos, (Fig. 5) aplica una corriente $\mathrm{I}_{\mathrm{o}}$ a través de dos electrodos y lee el valor del potencial de dos electrodos distintos. Este método consigue eliminar la impedancia de los electrodos siempre y cuando tengan una impedancia pequeña en comparación con la impedancia de entrada del circuito utilizado para detectar la tensión $\mathrm{V}_{\mathrm{o}} \mathrm{y}$ no provoquen una saturación de los circuitos que inyectan la corriente $\mathrm{I}_{\mathrm{o}}$. 
El método de los 3 electrodos, (Fig. 6) aplica una corriente $\mathrm{I}_{\mathrm{o}}$ a través de dos electrodos: uno inyector de corriente $\left(\mathrm{Z}_{\mathrm{e} 1}\right)$ y uno de referencia $\left(\mathrm{Z}_{\mathrm{e} 2}\right)$. El voltaje $\left(\mathrm{V}_{\mathrm{o}}\right)$ se detecta entre un tercer electrodo $\left(Z_{e} 3\right)$ y el de referencia $\left(\mathrm{Z}_{\mathrm{e} 2}\right)$. La diferencia de tensión medida corresponde a la caída de tensión provocada por la corriente que se aplica a la impedancia de interés $\left(Z_{\mathrm{x}}\right)$ y el segundo electrodo $\left(Z_{\mathrm{e} 2}\right)$.

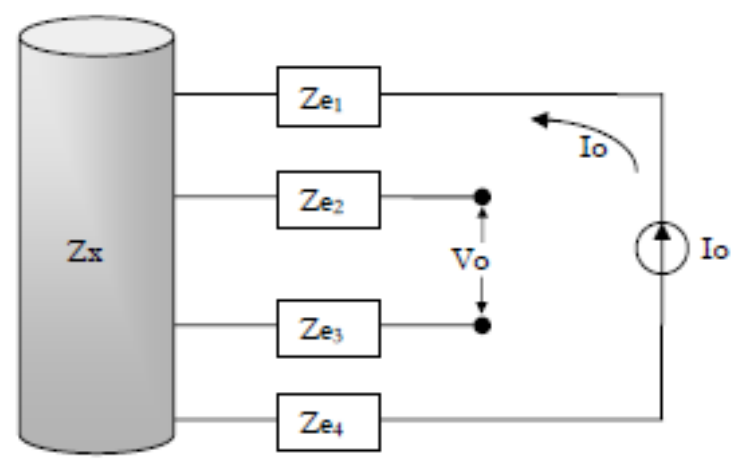

Fig. 5. Configuración del método a 4 electrodos (Salazar ,2004)

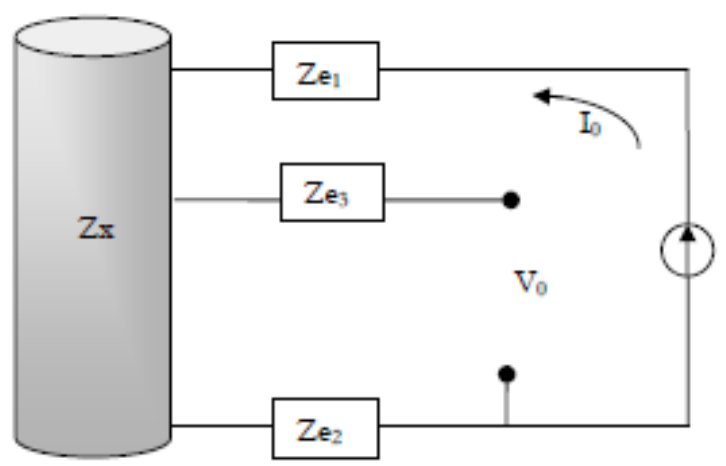

Fig. 6. Configuración del método a 3 electrodos (Salazar, 2004)

El método a 3 electrodos al ser sensible a la impedancia del electrodo $2\left(\mathrm{Z}_{\mathrm{e} 2}\right)$ y a la impedancia de la piel bajo este electrodo ha sido utilizado para medir la hidratación de la piel (Martinsen et al., 1995), para caracterizar electrodos (Riu et al., 1991) y en la 
calibración de sistemas de impedancia eléctrica (Bragos et al., 1999).

\subsection{Método sin Contacto basado en Inducción Magnética}

El método por inducción magnética se basa en la medición de la corriente circulante en los materiales conductores cuando son sometidos a un campo magnético o eléctrico. Las corrientes producen un campo magnético que puede ser detectado por un sistema receptor diseñado apropiadamente. Este método se ha verificado teórica y experimentalmente y ha sido utilizado en la inspección de piezas construidas con buenos conductores eléctricos (Gronhaug, 1988).

En la literatura se encuentran diferentes estructuras y maneras para disponer las bobinas (una o más) con respecto al medio (Blackwell, 1990). Algunas utilizan la misma espira como excitadora y sensora registrando los cambios que se producen en los parámetros (inductancia, frecuencia de resonancia, etc.) provocados por la presencia del medio analizado (Hagmann, 1993). En ocasiones se introduce una tercera bobina con el fin de anular el efecto del campo principal y dejar así solo la perturbación (señal a medir) (Gandhi \& Chen, 1990), en otras se introducen una cuarta o quinta bobina para lograr una mejor cancelación entre las bobinas sensoras (Hill \& Walsh, 1985).

La ausencia de contacto físico entre los elementos de medición y la muestra elimina los problemas de la interfaz electrodo medio, genera más confianza en cuanto a la seguridad eléctrica para las aplicaciones in vivo (Hagmann, 1993) y presenta más ventajas para mediciones en regiones rodeadas con medios de muy poca conductividad (como el cráneo) y en superficies irregulares (cicatrices o quemaduras) (Hagmann, 1993; Gandhi \& Chen, 1990). Como desventajas del este sistema se encuentra, la presencia de acoplamientos capacitivos y la dificultad de registro de señales muy pequeñas.

Los sistemas que crean imágenes a partir de las corrientes inducidas, así como la tomografía por inducción magnética, son completamente sin contacto. Los cambios en las densidades de corriente interna son medidos por bobinas en lugar de electrodos, 
aunque extremadamente sensibles a las mediciones de ruido (Katz et al., 2006).

\subsection{Métodos Híbridos}

En este método las corrientes son inducidas dentro del cuerpo por una o más bobinas circundantes y los electrodos superficiales son usados para la medición del potencial. La separación entre el suministro de corriente y el dispositivo medidor de voltaje conlleva beneficios como (Gencer \& Ider, 1994; Zlochiver et al., 2002):

- Control efectivo de la densidad de corriente al punto de interés mediante el ajuste de las bobinas.

- Aplicación de densidades de corriente mayores debido a la ausencia de una interfaz electrodo-piel.

- Alcance de la corriente a tejidos más profundos aún con la interferencia de tejidos aislantes tales como el cráneo.

- Mayor seguridad debido a la ausencia de contacto directo entre la fuente de corriente y el cuerpo del paciente.

Las técnicas híbridas no han sido estudiada tan extensamente en aplicaciones biomédicas de monitoreo e imagen como las corrientes inyectadas. Purvis et al., (1993) desarrolló un sistema experimental de 3 bobinas y 16 electrodos con frecuencia simple de $50 \mathrm{kHz}$. El sistema fue usado para estudiar la capacidad de imágenes de conductividad y permitividad de un phantoma circular, en el cual varias perturbaciones fueron introducidas (Purvis et al., 1993; Freeston \& Tozer, 1995; Scaife et al., 1994).

En otro sistema, fue estudiado el ruido como función del número de bobinas encontrando un incremento en los requerimientos del sistema al incrementar el número de bobinas. También fue evaluada como influía la forma de bobinas y la geometría en el rendimiento de imágenes, encontrando mejores resultados para las bobinas de radios grandes y más cercanas al objeto de medición (Gencer et al., 1996).

Una aplicación potencial de corrientes inducidas para la medición de bioimpedancia sería la evaluación de la densidad mineral de huesos (DMH) para seguimiento de la osteoporosis (Katz et al., 2006). La medida de DMH es la principal herramienta para la prevención de la osteoporosis, su diagnóstico y 
tratamiento. Algunas investigaciones han utilizado especímenes de hueso humanos y de animal, encontrando una fuerte correlación lineal entre la permitividad relativa del tejido del hueso y muchas de sus características mecánicas especialmente la DMH (Sierpowska et al., 2003). Esto llevó a reconocer que las propiedades dieléctricas podrían ayudar a predecir la calidad y los esfuerzos del hueso (Williams \& Saha, 1996; Sierpowska et al., 2003). La técnica de bioimpedancia propone una alternativa relativamente económica al comparar con otras técnicas existentes, sin radiación ionizante lo que la hace muy adecuado para seguimiento a largo plazo (Katz et al., 2006).

En cuanto a la creación de modelos matemáticos o computacionales, también han sido reportados modelos de simulación de corrientes inducidas. Los métodos más comunes de cálculo han sido el método de elementos finitos y el método de volumen finito. Estos métodos han sido utilizados para inyección y monitoreo de corrientes inducidas y para aplicaciones en imágenes (Abboud et al., 1994; Zlochiver et al., 2002; 2005).

\section{CARACTERÍSTICAS DE LA SEÑAL APLICADA}

La medición de la bioimpedancia eléctrica de tejidos biológicos y la demodulación de las señales de respuesta bio-modulada compleja, demandan inyectar excitaciones AC (estímulos) a través del tejido o través de los biomateriales bajo observación (Grimnes \& Martinsen, 2000). Las formas de onda de la excitación pueden ser diferentes y el procesamiento de las señales puede ser digital o análogo (Grimnes \& Martinsen, 2000; Pallas \& Webster, 1993).

La excitación con onda sinusoidal es preferible para dejar los errores de medición dentro de un rango aceptable. Sin embargo, la generación de señales de onda seno puras es más complicada y consume más potencia que la generación de algunas clases de ondas rectangulares (Gillian et al., 2004). Otra desventaja de la señal rectangular simple de dos niveles es el impacto de altos armónicos en la señal demodulada que genera como resultado errores de medición que dependen de la fase del vector de impedancia. 
Una consideración importante en el campo de la bioimpedancia es la frecuencia usada para la corriente de excitación. Los parámetros eléctricos del tejido, tales como la conductividad y la permitividad e incluso el potencial generado, son dependientes de la frecuencia (Katz et al., 2006). La expresión Ratio $\equiv 2 \pi f \varepsilon_{(f)} / \sigma_{(f)}$ representa la relación entre el desplazamiento (capacitivo) y la corriente conductiva. La influencia de los cambios de permitividad en los potenciales de superficie amplía la relación provocando que los cambios de permitividad representen cambios significativos en la corriente inducida al cuerpo. Esta relación es relativamente alta para bajas frecuencias $(f<100 \mathrm{~Hz})$ o altas frecuencias $(f>100 \mathrm{kHZ})$ pero pequeña en el rango de frecuencia intermedia $(100 \mathrm{~Hz}<\mathrm{f}<100 \mathrm{kHz})$.

Un estudio realizado por Williams \& Saha (1996) investigando en material óseo encontró la permitividad del hueso como una función de la densidad de este en tres frecuencias diferentes. Kartz et al., (2006) en su trabajo sobre tejido óseo, estableció que aunque las mediciones a muy bajas frecuencias llegarían a satisfacer muchos de los requerimientos, se encontrarían superpuestas con la actividad eléctrica de las fuentes biológicas endógenas, de donde una frecuencia alta combinada con una gran densidad de corriente arrojaría mejores mediciones. El valor seleccionado por Kartz et al., para las pruebas fue $1 \mathrm{~A}$ a $20 \mathrm{kHz}$ para la corriente de excitación de la bobina con una sola vuelta.

Algunas fuentes de error encontradas incluyen la posible polarización del gel conductor, la distancia hasta el dispositivo de medida o interferencias del gel o el cable de conexión, entre otras (Gillian et al., 2004; Morucci et al., 1996; Steendijk, 1993).

\section{APLICACIONES DE LA MEDICIÓN DE BIOIMPEDANCIA: CARACTERIZACIÓN DE TEJIDOS}

Normalmente el uso de la tomografía de impedancia eléctrica en seres humanos utiliza valores de corriente entre 0,1 y $10 \mathrm{~mA}$ y un rango de frecuencias desde $100 \mathrm{~Hz}$ a $1 \mathrm{MHz}$ (Paavle et al., 2007). Este rango está ligado a la preservación de la integridad del paciente estimulado y a minimizar los problemas de polarización 
en los electrodos. De manera más específica y para el uso de la ecuación de Cole-Cole, algunos estudios recomiendan las frecuencias de medición entre $100 \mathrm{~Hz}$ y $1 \mathrm{kHz}$ para una mejor estimación de $\mathrm{R}_{0}$, incluir frecuencias por debajo de $1 \mathrm{kHz}$ para $\mathrm{R}_{\infty} \mathrm{y}$ para $\tau$ las frecuencias de la onda de excitación deberán estar equidistantemente espaciadas en el lugar de Nyquis (Gabriel et al., 1996).

El método de medición denominado espectrografía de impedancia eléctrica, utiliza cuatro electrodos para el registro de voltaje y corriente a diferentes frecuencias de barrido para obtener los parámetros de la ecuación de impedancia de Cole-Cole (Curtze et al., 2004; Miron et al., 2002). Algunas de las aplicaciones que implementa esta técnica es el monitoreo de eventos respiratorios y composición corporal (Northrop, 2002), otros se describen en (Salazar, 2004; Beckmann et al., 2007; Tsunami et al., 2004; Kun \& Peura, 1999; Min et al., 2004; Rafiei et al., 2007). El método de espectroscopia comprende la evaluación intra y extracelular mediante la examinación del contenido de agua, grasa, hueso, músculo, etc., para alguna región particular del cuerpo (Evangelisti \& Talluri, 1994; Matthie et al., 1992). Materiales con mayor o menor cantidad de estos componentes determinan el comportamiento de las propiedades eléctricas, así por ejemplo la sangre o los músculos tienen una alta conductividad en comparación con los huesos o la grasa (Gabriel et al., 1996).

La tomografía también es usada como método de impedancia eléctrica (Northrop, 2002). Esta técnica realiza un corte tomográfico cuyos parámetros eléctricos son obtenidos a partir de un numero finito de electrodos ubicados alrededor de la periferia del objeto de interés, algunos ejemplos de estas aplicaciones se muestran en (Zapata et al., 2000; Rafiei et al., 2007).

El uso de la configuración de 4 electrodos para la medida de impedancia eléctrica ha sido también utilizado para monitorizar eventos del sistema respiratorio para el que se sugieren electrodos estables, con buena adherencia, baja impedancia de contacto y un área total grande (Mayotte et al., 1994). Una extensión del método a 4 electrodos es la utilización de 8, 16 o más electrodos no invasivos para obtener imágenes de impedancia distribuida (Eyuboglu et al., 1989). Sin embargo, el uso de múltiples 
electrodos (8 o más), hace que sea una técnica de difícil uso en la práctica clínica ya que requiere una colocación muy precisa de los electrodos (Frerichs, 2000; Cornish et al., 1999; Petrova, 1999; Luo et al., 1992). Kerner et al., (2002) utilizó 16 electrodos para obtener imágenes de cáncer de mama (basados en la diferencia morfológica del tejido canceroso) y uno de los primeros investigadores fue Jossinet (1988), quien realizó importantes experimentos ex vivo con tejido de mama a pesar de no haber reportado imágenes in vivo.

\section{MEDICIONES CLÍNICAS}

La influencia inminente de la posición del cuerpo en las mediciones de bioimpedancia fue reconocida hace algunos años por Kushner et al., (1996). En sus estudios mostró que la posición del cuerpo refleja un cambio continuo en la variable eléctrica medida que es dependiente del tiempo y que parece no tener un punto de estabilización después de 40 minutos en la misma posición. Con el fin de disminuir esta variable, se ha recomendado que el paciente permanezca en posición acostada y luego de cinco minutos en esta posición sea realizada la medición. Esta recomendación ha llegado a ser un procedimiento estándar (Stand 2/02.06.; Hydra ECF/ICF, 2004). Sin embargo, no es claro cuánto de esta combinación de tiempo y posición compensa los largos períodos de estar de pie o sentado.

De acuerdo con algunos autores la medición es afectada no solo por la posición del cuerpo, sino además por el momento del día en que se realice la medición (Medrano, 2007; Slinde \& Rossander, 2001; Slinde et al., 2003). Medrano en su trabajo mostró una reducción continua del valor de impedancia para un paciente pasando de estar sentado a estar de pie. Mediciones realizadas sobre personas que pasaban una gran parte del día en cama, mostraron un incremento en el comportamiento de la impedancia (Slinde et al., 2003). De estas mediciones se sospecha que entre más tiempo la persona haya estado sentada o parada, más tiempo será necesario para compensar el cambio de fluido (Medrano et al., 2006). 
Otra de las consideraciones a la hora de medir bioimpedancia es remover la capa de células muertas de la epidermis o al menos una parte de ellas, pues presentan características de alta impedancia que pueden producir errores en la medición. Algunos métodos de eliminación son: frotamiento con algún papel mojado en alcohol o acetona, abrasión con papel rugoso o simplemente frotar la zona con algún cepillo (Medrano, 2007).

Una muy importante recomendación para las mediciones superficiales es el uso de medio electrolítico entre la piel y el electrodo para evitar la interferencia de tejido muerto, el vello también afecta la interface electrolito-piel generando mal contacto y provocando mediciones erróneas (Salazar, 2004).

Este estudio en conceptos y trabajos desarrollados en la técnica de medición de bioimpedancia, permitió a los investigadores desarrollar un protocolo de medición y caracterización de condiciones necesarias para aplicar en pacientes la medición de impedancia utilizando un dispositivo medidor que se encuentra en su etapa final de desarrollo.

\section{REFERENCIAS}

Abboud, S., Eshel, Y., Levy, S., Rosenfeld, M., (1994); Numerical calculations of the potential distribution due to dipole sources in a spherical model of the head. Comput. Biomed. Res. 27:441- 455.

Beckmann, L., Riesen, D., Leonhardt, S., (2007); Optimal electrode placement and frequency range selection for the detection of lung water using Bioimpedance Spectroscopy. Proceedings of the 29th Annual International Conference of the IEEE EMBS Cité Internationale, Lyon, France August 23-26, FrB06.4.

Blackwell, (1990); The personal current meter-A novel ankle-worn device for the measurement of RF body current in a mobile subject. J. Radiol. Prot. UK, vol. 10, pp 109-114.

Bourne, J.R., Morucci, J.P., Valentinuzzi, M.E., Rigaud, B., Felice, C.J., Chauveau, N., Marsili, P.M., (1996); Bioelectrical Impedance Techniques in Medicine, Critical Reviews in Biomedical Engineering, Vol. 24, Issues 4-6. 
Bragos, R., Casas, O., Rosell, J., Warrem, M., Tresanchez, M., Carreño, A., Rodriguez-Sinovas, A., Cinca, J., (1999); In vivo detection of healed miocardium scar in pigs measuring the electrical impedance spectrum with an intracavitary catheter. 5th conference of the European Society for Engineering and Medicine, Barcelona Spain, pp. 487-488.

Cornish, B.H., Jacobs, A., Thomas, B.J. and Ward, L.C. (1999); Optimizing electrode sites for segmental bioimpedance measurements. Physiological Measurement, 20, 241-250.

Curtze, S., Dembo, M., Miron, M., Jones, D., (2004); Dynamic changes in traction forces with DC electric field in osteoblast-like cells. Journal of Cell Science, 117, 2721-2729.

Evangelisti, A., Talluri, T., (1994); Bioelectric impedance analysis (BIA) cluster analysis. Proceedings of the 2-nd Con Mechatronics'9.1 Poland, Sept. 22-23, 187-190.

Eyuboglu, B.M., Brown, B.H. and Barber, D.C. (1989); In vivo imaging of cardiac related impedance changes. Engineering in Medicine and Biology Magazine, IEEE, 8, 39-45.

Foster, K.R., Schwan, H.P., (1996); Dielectric properties of tissues. In Handbook of Biological Effects of Electromagnetic Fields. C. Polk and E. Postow, editors. CRC Press Inc., Boca Raton, FL. 25-102.

Fresenius Medical Care, (2006); Body Composition Monitor (BCM). Operating instructions Fresenius Medical Care. Stand 2/02.06.

Freeston, I.L., Tozer, R.C., (1995); Impedance imaging using induced currents. Physiol. Meas. 16:A257-A266.

Gandhi, O., Chen, J., (1990); Meters for assessment of induced body and contact current and store energy in electromagnetic fields. Bioelectromagn. Soc. Twelfth Annu. Meet. Abstracts. San -Antonio, TX, pp. 35-36.

Frerichs, I., (2000); Electrical impedance tomography (EIT) in applications related to lung and ventilation: a review of experimental and clinical activities. Physiological Measurement, 21, R1-R21.

Gabriel, S., Lau, R.W, Gabriel, C., (1996a); The dielectric properties of biological tissues: II. Measurements in the frequency range $10 \mathrm{~Hz}$ to 20 GHz. Phys. Med. Biol. 41, 2251-69.

Gabriel, S., Lau, R.W, Gabriel, C., (1996b); The dielectric properties of biological tissues: III. Parametric models for the dielectric spectrum of tissues. Phys. Med. Biol. 41, 2271-93. 
Geddes, L.A., (1997); Historical evolution of circuit models for the electrode-electrolyte interface. Ann. Biomed. Eng. 25, 1-14.

Gencer, N.G., Ider, Y.Z., Williamson, S.J., (1996); Electrical impedance tomography: induced-current imaging achieved with a multiple coil system. IEEE Trans. Biomed. Eng. 43:139-149.

Gencer, N.G., Kuzuoglu, M., Ider, Y. Z., (1994); Electrical impedance tomography using induced currents. IEEE Trans. Med. Imaging 13:338-350.

Gencer, N.G., Ider, Y.Z., (1994); A comparative study of several exciting magnetic fields for induced current EIT. Physiol. Meas. 15:A51-57.

Gillian, Y., Edith, D., Gurewitsch, Shaahinfar, A., Elbert, S., Sampattavanich, S., Ruffner, M., Ching, K., Allen, R., (2004); Measuring Bioimpedance in the Human Uterine Cervix: Towards Early Detection of Preterm Labor. Proceedings of the 26th Annual International Conference of the IEEE EMBS San Francisco, CA, USA - September 1-5.

Gonzalez, C., Gonzalez, C.H., Ramos, A., (2007); Best published equation for the calculation of Body Fat in a sample of Colombian young males using Bioelectrical Impedance. Analysis Alemania, IFMBE Proceedings, vol:17 fasc: 1 págs: $803-806$.

Gonzalez, C.; Brown, B., Tidy, J., Bremmer, J., (2006); Impedance spectral measurements made through a membrane infection barrier. En: Gran Bretaña Medical \& Biological Engineering \& Computing ed: Springer v.44 fasc. 12 p.1085 - 1091 .

Gonzalez, C., Padilla, L., Zuñiga, O., (1997); Detection of animal tissue thicknes using simple vertical electric sounding (VES). En: Gran Bretaña Physiological Measurement, ed:v.18 fasc. p.85 - 91.

Grimnes, S., Martinsen, Ø.G., (2000); Bioimpedance and Bioelectricity Basics. London, UK: Academic Press.

Gronhaug, K., (1988); Measurement of EMP induced currents in the human body. FFI/NOTAT-88/4038, Norwegian Defence Res.

Hagmann, M., (1993); Noninvasive measurement of current in the human body for electromagnetic dosimetry. IEEE transactions of biomedical engineering, vol. $40 \mathrm{~m}$, No. 5 .

Hill, D., Walsh, J., (1985); Radiofrequency current through the feet of a grounded human, IEEE Trans. Electromag. Compart., Vol. 27, pp. 1823. 
Hydra ECF/ICF; Xitron Technologies-Model 4200, (2001); Bioimpedance Spectrum Analyser. Operating Manual, Xitron Technologies Inc. San Diego, CA.

Jossinet, J. (1988); A hardware design for imaging the electrical impedance of the breast. Clinical physics and physiological measurement, 9, 25-28.

Katz, S., Zlochiver, S., Abboud, S., (2006); Induced Current Bio-impedance Technique for Monitoring Bone Mineral Density-A Simulation Model. Annals of Biomedical Engineering, Vol. 34, No. 8, August pp.1332-1342.

Kerner, T.E., Paulsen, K.D., Hartov, A., Soho, S.K. and Poplack, S.P. (2002); Electrical impedance spectroscopy of the breast: clinical imaging results in 26 subjects. Medical Imaging, IEEE Transactions on, $21,638-645$.

Kun, S., Peura, R.A., (1999); Selection of measurement frequencies for optimal extraction of tissue impedance model parameters. Med. Biol. Eng. Comput., 37, 699-703.

Kushner, R., Gudivaka, R., Schoeller, D., (1996); Clinical characteristics influencing bioelectrical impedance analysis measurements; Am. J. Clin. Nutr., vol. 64(supl), 423-427.

Luo, S., Afonso, V.X., Webster, J.G. and Tompkins, W.J. (1992); The Electrode System in Impedance-Based Ventilation Measurement. IEEE Transactions on Biomedical Engineering, 39, 1130-1141.

Martinsen, O.G., Grimnes, S., Mirtaheri, P., (2000); Non-invasive measurement of postmortem changes in dielectric properties of haddock muscle - a pilot study. J Food Eng, 43, 189-192.

Martinsen, O.G., Grimnes, S., Karlsen, J., (1995); Electrical Methods for skin moisture assessment. Skin Parmacology., 8, 237-245.

Matthie, J.R., Withers, P.O., Loan, M.D., Mayclin P.L. (1992); Development of the commercial complex bioimpdance spectroscopic system for determining intracellular water and extracellular water volumes. Proceedings of VUIth Int. Con. Electr. Bio-Impedance. Finaland, July 28-31.

Mayotte, M.J., Webster, J.G. and Tompkins, W.J. (1994); A Comparison of Electrodes for Potential Use in Pediatric Infant Apnea Monitoring. Physiological Measurement, 15, 459-467. 
Medrano, G., (2007); Modeling the Influence of Body Position in Bioimpedance Measurements. Proceedings of the 29th Annual International Conference of the IEEE EMBS. Cité Internationale, Lyon, France. August 23-26.

Medrano G., Beckmann, L., Leonhardt, S., (2006); Einfluss der Koerperlage auf Bioimpedanz-Spektroskopie Messungen. Presented at the Gemeinsame Jahrestagung der Deutschen, Oesterreichischen und Schweizerischen Gesellschaft fuer Biomedizinische Technik, ETH Zuerich, 6 - 9 Sept.

Min, M., Land, R., Märtens, O., Parve, T., Ronk, A., (2004); Sampling Multichannel Bioimpedance Analyzer for Tissue Monitoring. Proceedings of the 26th Annual International Conference of the IEEE EMBS San Francisco, CA, USA, September 1-5, 902-905.

Miron, M., Curtze, S., Jones, D.B., (2002); Force Distributions Across Osteoblasts Caused by Electric Fields Produce Actin Disruption, Remodeling and Orientation: A mechanism Regulated by Calcium. Presented at the 21th Annual Meeting of the Society for Physical Regulation in Biology and Medicine. January 30-February 1, San Diego, California, USA.

Moncada M.E., Cadavid, H., Pinedo, C.R., Martínez, A., (2008); Desarrollo e implementación de una técnica para la medición de impedancia en muestras de hueso húmedo de bovino. Revista Facultad De Ingenieria Universidad De Antioquia, v.44 fasc. 1 p.75 - 82.

Morucci J.P., Valentinuzzi M.E., Riguard B, Felice C.J., Chaveau N, Marsili P.M., (1996); Bioelectrical Impedance Techniques In Medicine. Critical Reviews in Biomedical Engineering, 24(4-6):223255.

Northrop, R., (2002); Noninvasive instrumentation and measurement in medical diagnosis. The biomedical engineering series editor: Michael R. Newman. CRC press LLC. Boca Raton London New York Washington, D.C.

Paavle, T., Annus, P., Kuusik. A., Land, R., Min, M., (2007); Bioimpedance Monitoring with Improved Accuracy Using Three-Level Stimulus. Circuit Theory and Design, ECCTD 2007. 18th European Conference on. Date:27-30 Aug. 412 - 415.

Pallas-Areny, R., Webster, J.G., (1993); Bioelectric impedance measurements using synchronous sampling, IEEE Trans. Biomed. Eng., vol. 40, no. 8, pp. 824-828. 
Petrova, G.I., (1999); Influence of electrode impedance changes on the commonmode rejection ratio in bioimpedance measurements. Physiological Measurement, 20, N11-N19.

Purvis, W.R., Tozer, R.C., Anderson, D.K., Freeston, I.L., (1993); Induced current impedance imaging. Proc. IEEE 140:135-141.

Rafiei-Naeini, M., Wright, P., McCann, H., (2007); Low-Noise Measurement for Electrical Impedance Tomography. ICEBI 2007, IFMBE Proceedings 17, 324-327.

Rigaud, B., Morucci, J.P., Chauveau, N., (1996); Bioelectrical impedance techniques in medicine. Bioimpedance measurement -Second section: Impedance spectrometry. Critical review in Biomedical Engineering, 24, 257-351.

Ragheb, T., Geddes, L.A., (1990); Electrical properties of metallic electrodes, Med Biol Eng Comput 28, pp. 182-186.

Riu, P., Rosell, J., Lozano, A., Pallas, R., (1992); A broadband system for multifrequency static imagine in electrical impedance tomography. Clin. Phys. Physiol. Meas. 13 Suppl. A:61-65.

Salazar, Y., (2004); Caracterización de tejidos cardíacos mediante métodos mínimamente invasivos y no invasivos basados en espectroscopia de impedancia eléctrica. Tesis doctoral. Universidad Politécnica de Cataluña. Programa de ingeniería biomédica.

Salazar, Y., Bragos, R., Casas, O. et al., (2004); Transmural Versus Nontransmural In Situ Electrical Impedance Spectrum for Healthy, Ischemic, and Healed Myocardium. IEEE Trans Biomed Eng 51, 1421-1427.

Scaife, J.M., Tozer, R.C., Freeston, I.L., (1994); Conductivity and permittivity images from an induced current electrical impedance tomography system. IEE Proc. Meas. Technol, 141(5):356-362.

Sierpowska, J., Toyras, J., Hakulinen, M.A, Saarakkala, S., Jurvelin, J.S., Lappalainen, R., (2003); Electrical and dielectric properties of bovine trabecular bone-relationship with mechanical properties and mineral density. Phys. Med. Biol. 48:775-786.

Slinde, F., Rossander-Hulthén, L., (2001); Bioelectrical impedance: effect of 3 identical meals on diurnal impedance variation and calculation of body composition”, Am J Clin Nutr., vol. 74, 474-478. 
Slinde F., Bark, A., Jansson, J., Rossander-Hulthén, L., (2003); Bioelectrical impedance variation in healthy subjects during $12 \mathrm{~h}$ in the supine position, Clinical Nutrition, vol. 22 (2), pp. 153-157.

Steendijk, P., et al., (1993); The Four Electrode Resistivity Technique in Anisotropic Media: Theoretical Analysis and Application on Myocardial Tissue in Vivo. IEEE Transactions on Biomedical Engineering 40.11, 1138-48.

Tsunami, D., McNames, A., Colbert, S., Pearson, R., Hammerschlag, (2004); Variable Frequency Bioimpedance Instrumentation. Proceedings of the 26th Annual International Conference of the IEEE EMBS San Francisco, CA, USA, September 1-5, 2356-2389.

Williams, P.A., Saha, S., (1996); The electrical and dielectric properties of human bone tissue and their relationship with density and bone mineral content. Annals. Biomed. Eng. 24:222-233.

Zapata, A., Gaona, A., Castro, M., Aguillón, M., Vázquez, D., (2000); Proyecto de tomografía por el método de impedancia eléctrica. Avances actuales. Salud Mental V. 23, No. 2.

Zhu, F., Kuhlman, M.; Kotanko, P., Handelman, G., Leonard, E.F., Levin, N.W., (2007); A Device for Monitoring Hydration State in Hemodialysis Patients Using a Calf Bioimpedance Technique. ICEBI 2007, IFMBE Proceedings 17, pp. 775-778.

Zlochiver, S., Rosenfeld, M. Abboud, S., (2005); Contactless bioimpedance monitoring technique for brain cryosurgery in a 3D head model. Annals. Biomed. Eng. 33:615-625.

Zlochiver, S., Radai, M., Rosenfeld, M., Abboud, S., Dong, X.Z., Liu, R.G., You, F. S., Xiang, H. Y., Shi, X.T., (2004); Induced current electrical impedance tomography system: experimental results and numerical simulations. Physiol. Meas. 25:239-255.

Zlochiver, S., Radai, M., Rosenfeld, M., Abboud, S., (2003); Induced current impedance tomography: A 2-D theoretical simulation. IEEE Trans. Med. Imaging 22(12).

Zlochiver, S., Radai, M., Rosenfeld, M., Abboud, S., (2002); Induced current impedance technique for monitoring brain cryosurgery in a two-dimensional model of the head. Annals. Biomed. Eng. 30:11721180 . 